Original Articles

\title{
Reduced sensitivity to social priors during action prediction in adults with autism spectrum disorders
}

\author{
Valerian Chambon ${ }^{\mathrm{a}, \mathrm{b}, *}$, Chlöé Farrer $^{\mathrm{c}}$, Elisabeth Pacherie ${ }^{\mathrm{a}}$, Pierre O. Jacquet ${ }^{\mathrm{d}}$, Marion Leboyer $^{\mathrm{e}}$, \\ Tiziana Zalla ${ }^{\mathrm{a}, *}$ \\ a Institut Jean Nicod, CNRS UMR 8129, Institut d'Etude de la Cognition, École Normale Supérieure E' PSL Research University, 29 rue d'Ulm, 75005 Paris, France \\ ${ }^{\mathrm{b}}$ Department of Neuroscience, Biotech Campus - University of Geneva, 1211 Geneva, Switzerland \\ 'Centre de Recherche Cerveau et Cognition, Université de Toulouse, UPS-CNRS, Place du Docteur Baylac, Pavillon Baudot, 31059 Toulouse, France \\ ${ }^{\mathrm{d}}$ Laboratoire Psychologie de la Perception, CNRS UMR 8242, Université Paris-Descartes, 45, rue des Saints-Pères, 75006 Paris, France \\ e INSERM U955, IMRB, University Paris Est Creteil, AP-HP, Henri Mondor-Albert Chenevier Hospitals, Department of Psychiatry, Fondation FondaMental, French National \\ Science Foundation, 40 rue de Mesly, 94000 Créteil, France
}

\section{A R T I C L E I N F O}

\section{Article history:}

Received 20 November 2015

Revised 6 December 2016

Accepted 20 December 2016

\section{Keywords:}

Mentalizing

Action prediction

Prior knowledge

Sensory evidence

Bayesian inference

Autism spectrum disorders

\begin{abstract}
A B S T R A C T
An extensive amount of evidence has documented a diminished ability to predict and understand other people's action in individuals with autism spectrum disorders (ASD). Recently, two theoretical accounts, the "Hypo-priors" and the "Aberrant precision" hypotheses, have suggested that attenuated Bayesian priors or an imbalance of the precision ascribed to sensory evidence relative to prior expectations may be responsible for the atypical perceptual experience and difficulties with action understanding in ASD. In the present study, we aimed to directly investigate whether difficulties in the appreciation of others' intentions can be accounted for by abnormal interaction between these two types of information: (i) the sensory evidence conveyed by movement kinematics, and (ii) the observer's expectations, acquired from past experience or derived from prior knowledge. To test this hypothesis, we contrasted the ability to infer Non-Social and Social intentions in adults with and without ASD, using a series of tasks in which both sensory evidence and prior expectations were manipulated. The results showed that attenuated effect of prior expectations in ASD individuals does not result from a generalized impairment in mentalizing, but one confined to social intentions. Attenuated priors in the social domain predicted the severity of clinical symptoms in the area of social interaction. Importantly, however, we found that reduced priors in the social domain could be compensated by ASD through observational learning, i.e. through deriving statistical regularities from observed behaviours. This capacity to balance reduced social expectations by learning inversely correlated with the severity of repetitive and stereotyped behaviours. Collectively, these findings suggest that adults with ASD exhibit a disturbance in the inferential mechanism that integrates sensory evidence into prior beliefs to produce accurate inferences about other people's intentions.
\end{abstract}

(C) 2016 Elsevier B.V. All rights reserved.

\section{Introduction}

Diminished social functioning constitutes one of the core features of ASD. In the last decades, an extensive literature has focused on disturbances of mentalizing or Theory of Mind (ToM), i.e. the ability to attribute beliefs and other mental states to oneself and to others, in individuals with ASD (e.g. Baron-Cohen, 1995; Baron-Cohen, Leslie, \& Frith, 1985, 1986; Frith, 1989; Happé \& Frith, 1996). However, the nature of the impairment affecting

* Corresponding authors at: Institut Jean Nicod, Ecole Normale Supérieure, 29 rue d'Ulm, 75005 Paris, France (V. Chambon).

E-mail addresses: valerian.chambon@ens.fr (V. Chambon), tiziana.zalla@ens.fr (T. Zalla) action and intention understanding in ASD is still a matter of debate (Hamilton, 2009). While children with autism have impaired or delayed maturation of ToM, adults with highfunctioning ASD continue to experience difficulties with understanding others' intentions in real-life situations, even when they succeed on standard tests for ToM. Questions have also been raised as to whether difficulties reflect selective impairments in mentalizing or disturbances of low-level mechanisms of action perception or of the resonance mechanism supported by the neuron mirror system (Smith \& Bryson, 1994; Hughes, 1996; Russell \& Jarrold, 1998, 1999; Zalla, Bouchilloux et al., 2006; Zalla, Labruyere, \& Georgieff, 2006; Cattaneo et al., 2007; Fabbri-Destro, Cattaneo, Boria, \& Rizzolatti, 2009; Vanvuchelen, Roeyers, \& De Weerdt, 
2007; von Hofsten \& Rosander, 2012; Zalla, Labruyère, \& Georgieff, 2013).

Using different experimental paradigms, current studies support the notion of preserved goal and action understanding in individuals with ASD (Hamilton, 2009) while difficulties arise when goals and actions are not visible outcomes, and have to be inferred on the basis of the available visual and contextual information (Cattaneo et al., 2007; Gomot \& Wicker, 2012; Zalla, Labruyere, Clément, \& Georgieff, 2010). These results suggest that difficulties with action understanding in individuals with ASD might arise from impairments in complementing (i.e. contextualizing) the available sensory evidence with other information. Recently, it has been suggested that sensory atypicalities in ASD - such as enhanced sensations, experience of sensory overload, or hypersensitivity - can be explained by a diminished influence of top-down prior expectations on perceptual experience ("hypo-priors") associated with increased reliance on sensory evidence, possibly as a consequence of enhanced "bottom-up" functioning (Lawson, Rees, \& Friston, 2014; Van de Cruys et al., 2014; see also Pellicano \& Burr, 2012). Such abnormal interplay between topdown priors and bottom-up sensory evidence may provide a simple explanation for ASD difficulties in inferring other people's intentions, by yielding significant deviations from normative Bayesian inference (McKay, 2012).

Bayesian models of intention understanding posit that our brain is constantly engaged in the process of drawing inference on the basis of two distinctive types of information: the sensory evidence conveyed by movement kinematics and the observer's prior expectations about which intention is the most likely cause of what is observed, given past experience (Baker, Saxe, \& Tennenbaum, 2009; Baker, Tenenbaum, \& Saxe, 2006; Chambon, Domenech et al., 2011; Kilner, 2011). Intention inference is contingent upon an adaptive interplay between these two sources of information, with observers tending to rely progressively more on their prior expectations as the reliability of sensory evidence decreases, and vice versa. Crucially, this interaction has also been found to vary according to the 'type' of intention to be inferred, with participant's prior experience gaining weight over sensory evidence when inferring motor intentions directed at a third party (Social intentions) rather than motor intentions directed at objects in isolation (Non-Social intentions) (Chambon, Domenech et al., 2011; Chambon, Pacherie et al., 2011).

Here, in accordance with theoretical models (Lawson et al., 2014; Pellicano \& Burr, 2012), we hypothesized that diminished mentalizing abilities in individuals with ASD could be accounted for by an abnormal weighting of these two types of information (prior expectations and sensory evidence), in turn depending on the type of intention to be inferred (Social vs. Non-Social intentions). Specifically, attenuated "top-down" priors might be responsible for difficulties encountered by ASD in inferring social intentions. Indeed, social intentions often show a complex relationship with the behaviour they cause, i.e., in social situations many competing intentions are potentially consistent with what is observed. As such, social intentions cannot be unambiguously inferred from mere observation, and prior knowledge is required to constrain the space of candidate intentions (Chambon, Domenech et al., 2011; Kilner, Friston, \& Frith, 2007a, 2007b). If individuals with ASD do show attenuated priors, one would expect deficits to be more severe in social situations where priors are massively required (Lawson et al., 2014). Thus, particularly relevant to the autistic symptomatology is the hypothesis that poor "socialspecific" priors may result in an incapability to reduce the intrinsic uncertainty of social behaviours, in turn compromising one's ability to effectively interact with others (Chambon, Pacherie et al., 2011; Sinha et al., 2014; see also Fletcher \& Frith, 2009). Although not yet supported by direct empirical evidence, this hypothesis is otherwise consistent with observations made in individuals with schizophrenia suffering from negative ("autistic-like") symptoms. While these patients exhibit poor prior expectations in the social domain, they also rely more strongly on sensory evidence to infer intentions when those are directed at a third party rather than at a non-meaningful object (Chambon, Pacherie et al., 2011). Interestingly, it has been suggested that, in these patients, social situations may not prompt the same expectations as those typically observed in comparison participants, leading to the formation of abnormal (unreliable) predictions about others' social intentions (Zalla, Verlut, Franck, Puzenat, \& Sirigu, 2004; Zalla, Bouchilloux et al., 2006; Zalla, Labruyere et al., 2006; Barbalat, Chambon, Franck, Koechlin, \& Farrer, 2009; Barbalat et al., 2011; Chambon, Pacherie et al., 2011; Chambon et al., 2012).

In the present study, we aimed to test this hypothesis by contrasting the ability to infer non-social and social intentions in a group of adults with ASD, as compared to a control group of typically developed adults. Participants were presented with a series of video clips showing an actor manipulating (either transporting or rotating) a non-meaningful object and asked to infer intentions directed at an object or intentions directed at a third party, respectively. In both the "Social" and the "Non-Social" intention tasks, the specific contributions of sensory evidence and prior expectations to the intentional inference were systematically manipulated by varying the amount of visuo-motor evidence conveyed by the action scene (i.e. the completeness of action sequences) and the probability of occurrence associated with each different intention. We then looked at (i) whether participants' performance on each condition could be accounted for by an abnormal dependence on visuo-motor evidence and/or prior expectations, depending on the type (Social vs. Non-Social) of the intention being manipulated, and (ii) whether this abnormal dependence - if observed - correlated with the severity of autistic symptoms, as measured by the Autism Diagnostic Interview-Revised (ADI-R) (Lord, Rutter, \& Le Couteur, 1994).

\section{Methods and materials}

\subsection{Participants}

Eighteen adult participants with ASD and twenty comparison participants (CP) were recruited to participate in the study from Albert Chenevier Hospital in Créteil. The groups were matched for age, education, gender and full-scale IQ as measured by the Wechsler Adult Intelligence Scale (Wechsler, 1999) (see Table 1). All participants were screened for exclusion criteria (dyslexia, epilepsy, and any other neurological or psychiatric conditions) prior to taking part to the study. Participants in the ASD group had a clinical diagnosis of Asperger Syndrome or High Functioning Autism

Table 1

Means (and standard deviations) of demographic and clinical data for participants with ASD and the comparison volunteers.

\begin{tabular}{llll}
\hline & ASD & Comparison & $p$ value \\
\hline N (male:female ratio) & $15: 3$ & $16: 4$ & 0.9 \\
Age in years & $35.7(7.7)$ & $34.8(6.4)$ & 0.7 \\
$\quad$ (mean, SD, range) & & & \\
$\begin{array}{l}\text { Education in years } \\
\quad \text { (mean, SD) }\end{array}$ & $14.3 \pm 3.3$ & $14.5 \pm 3.4$ & 0.28 \\
ADI [B,C,D] & $14.6(5.7) ; 9.2(5.2) ;$ & - & \\
$\quad 6.9(3.2)$ & & \\
Full-scale IQ & $104.9(18.6)$ & $107.7(7.9)$ & 0.55 \\
$\quad$ Verbal IQ & $108.6(15.6)$ & $109.7(7.8)$ & 0.78 \\
Performance IQ & $98.7(20.8)$ & $103.7(8.1)$ & 0.35 \\
\hline
\end{tabular}

$[B]=$ reciprocal social interaction, $[C]=$ communication, $[D]=$ stereotyped behaviours. 
(HFA/AS) according to DSM-IV-TR (American Psychiatric Association, 2000) and ASDI (Asperger Syndrome Diagnostic Interview, Gillberg, Gillberg, Råstam, \& Wentz, 2001) and the ADOS (Lord et al., 2000) by experienced clinicians. Semi-structured interview with parents or caregivers using the ADI-R (Autism Diagnostic Interview, Lord et al., 1994) yielded scores in three content areas: [B] social interaction, [C] communication, and [D] repetitive and stereotyped behaviours, allowing the separate quantification of severity of the symptomatology. The cut-off points for these domains were 10,8 , and 3 , respectively. All participants scored above the cut-off points (Table 1). All participants gave informed consent to take part in the study. The present research has been approved by the local Ethical committee (Inserm, Institut Thématique Santé Publique; C07-33) and performed in accordance with the ethical standards laid down in the 1964 Declaration of Helsinki.

\subsection{Experimental design}

\subsubsection{General procedure}

Both Social and Non-Social intention tasks were adapted from Chambon, Domenech et al. (2011) and required the participants to infer the intention of one or two actors manipulating nonmeaningful objects. Each task consisted of six blocks, and each block consisted of two experimental phases: an induction phase, followed by a testing phase (see Fig. 1, and Supporting information Fig. S1). The induction phase consisted of 12 action sequences conveying a very high amount of visuo-motor evidence (1880 ms after movement onset) to allow the participants to clearly distinguish the different intentions being enacted. The testing phase consisted of 36 interleaved trials in which action sequences were shortened to convey various amounts of visuo-motor evidence (low, moderate, or high - i.e., 1480, 1560, and $1640 \mathrm{~ms}$ after movement onset, respectively; see Chambon, Domenech et al., 2011, Supporting information - Text S1, for the selection and control of these amounts). Each block was thus defined by one induction phase (12 trials) directly followed by a testing phase (36 trials) - i.e., 48 trials in total. In each block of both the Non-Social and the Social tasks, prior expectations were manipulated by setting the probability of occurrence of one intention to 0.66 (i.e., $66 \%$ of the trials) while setting the probability of the competing intention to 0.33 (i.e., 33\% of the trials). Accordingly, the intention with the greatest probability of occurrence was labelled the likely intention, whereas the intention with the lowest probability of occurrence was labelled the unlikely intention. This bias was randomly assigned so that each type of intention was equally biased across participants. Each video clip was presented only once to prevent any influence of memorized kinematic parameters on participants' performances ( 72 unique videos per intention and amount of sensory evidence). The order of the two tasks (Social and Non-Social) was counterbalanced across participants.

Video clips were recorded using a digital camera (Sony ${ }^{\circledR}$ - HDRSR7) and were tailored using the software Adobe Premiere ${ }^{\circledR}$. Stimuli were display on a screen at a frame rate of $60 \mathrm{~Hz}$ using Presentation $^{\circledR}$ software (Neurobehavioral Systems, www.neurobs.com). All the "Non-Social" video clips were performed by the same actor, and only featured her naked arm. All the "Social" video clips were performed by the same two actors and only featured their naked arms.

\subsubsection{Non-Social intention task}

Video clips depicted an actor's naked arm manipulating (rotating or transporting) a rectangular cube (Fig. 1A, 'Non-Social task'). A trial started with a fixation point at the centre of the screen for 500-2500 ms. Once the fixation disappeared, a video clip showing a resting hand positioned in front of a cube $(1000-1500 \mathrm{~ms}$; 'preparation' phase), followed by a reaching-and-grasping movement aiming at either rotating or transporting the cube

\section{A. Non-Social}

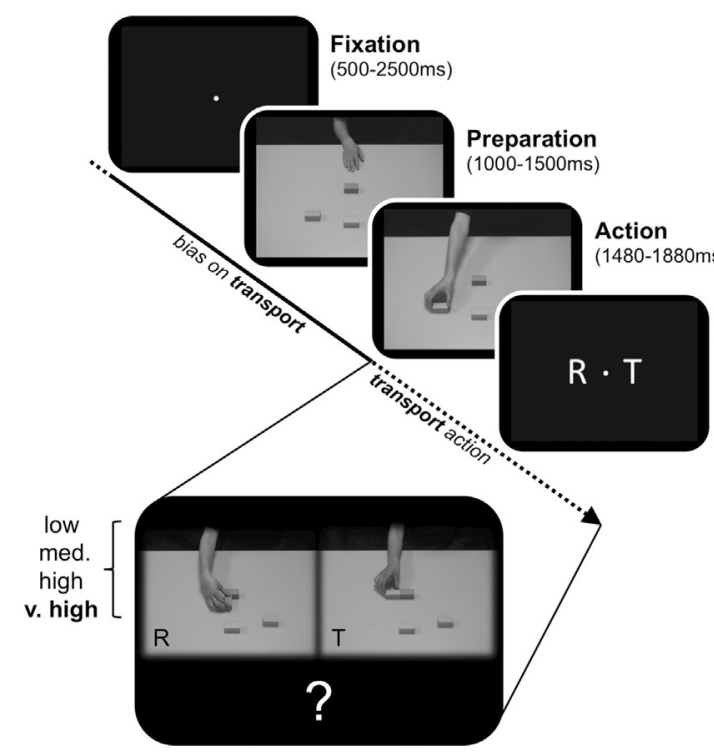

B. Social

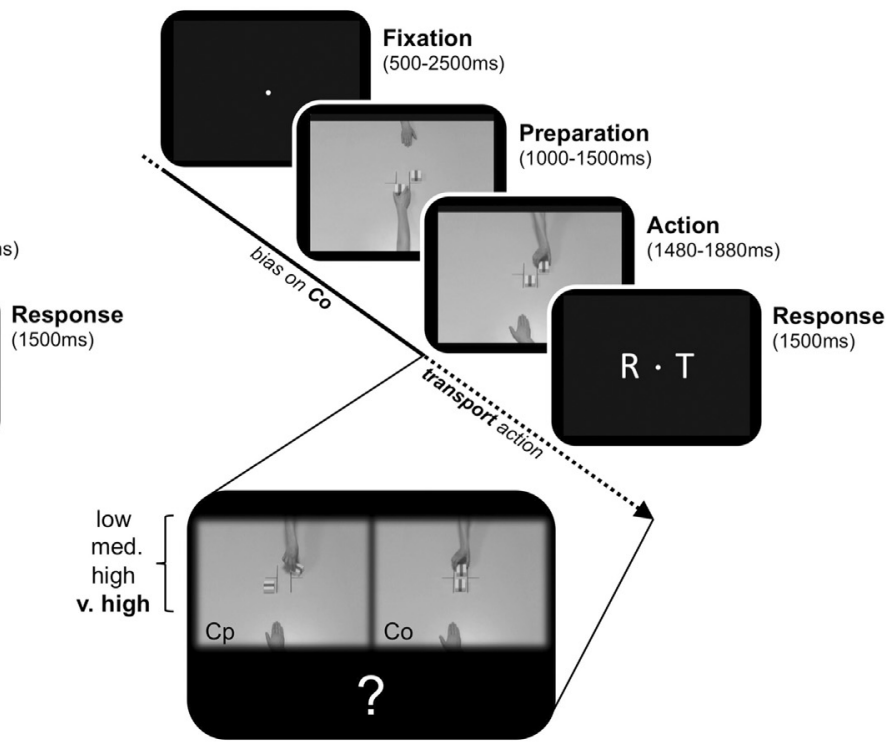

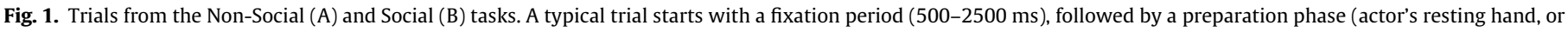

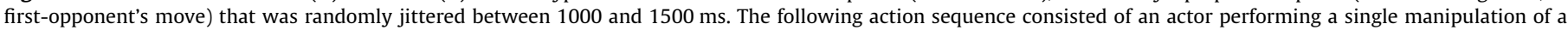

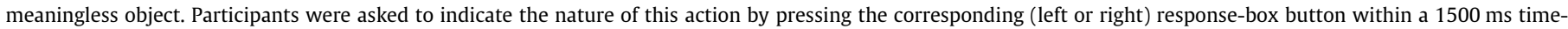

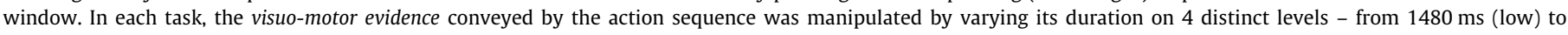

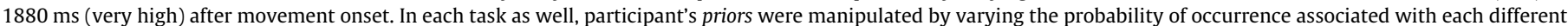

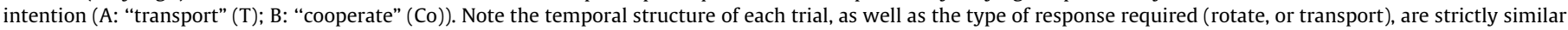
across both tasks. 
(1480-1880 ms; 'action' phase), was displayed. After a delay (central fixation point on a black screen displayed for 500-1000 ms, uniformly jittered), two letters ( $\mathrm{T}$ for 'transporting', $\mathrm{R}$ for 'rotating') were randomly displayed on the left and right sides of a central fixation dot. Participants indicated their belief over the actor's intention by pressing the corresponding response button as quickly and accurately as possible (left or right, time limit: $1500 \mathrm{~ms}$ ). Once a response was given, the next trial started. The Non-Social motor intention for which the probability of occurrence was set to 0.66 (i.e., the likely intention) was counterbalanced across participants.

\subsubsection{Social intention task}

Video clips depicted two actors manipulating (rotating or transporting) rectangular cubes (Fig. 1B, 'Social task'). In this task, participants were instructed to infer whether a social intention was of either a cooperative or defective nature. Participants observed two actors engaged in a social game, in which they either cooperated by coordinating their actions in order to achieve a shared goal, or defected by refusing to coordinate their actions. Taking turns, the actors could either transport the closest cube to the middle column of a 3-by-2 grid (cooperation), or rotate it so that it stayed in place (defection). Trials in the Social condition had the same overall structure as Non-Social trials: the first actor's action was entirely disclosed to the participants, whereas the second actor's action was made incomplete by varying the video clip duration across the trials $(1480,1560,1640$, or $1880 \mathrm{~ms}$ after onset of the second actor's action). After a delay (central fixation point on a black screen displayed for 500-1000 ms, uniformly jittered), two letters ( $\mathrm{T}$ for 'transporting', $\mathrm{R}$ for 'rotating') were randomly displayed on the left and right sides of a central fixation dot. Participants indicated their belief over the actor's social intention (i.e. cooperation or defection) by pressing the corresponding response button as quickly and accurately as possible (left or right, time limit: $1500 \mathrm{~ms}$ ). Note that the second actor's social intention either differed from that of the first actor (i.e., the first actor defected and the second cooperated, or the first actor cooperated while the second defected) or it mirrored the first actor's intention (i.e., both actors cooperated or defected). This second type of response strategy is known as a "tit-for-tat" (TFT) strategy. All over the session, the probability that the second actor responded tit-for-tat was therefore set to 0.66 so that, on average, she was more likely to cooperate (rather than defect) if the first actor had previously cooperated, and to defect (rather than cooperate) if the first actor had previously defected (see Supplementary Methods, 'Social intention task', for further details).

\section{Results}

\subsection{Discriminability index $\left(d^{\prime}\right)$ and response bias (c)}

Two types of analyses were conducted. Correct Response rates (CR) were first analysed within each group using a $2 \times 2 \times 4 \times 2$ repeated-measures ANOVA with Group (Comparison versus ASD) as a between-subjects factor, and type of Intention (Non-Social versus Social), Amount of visual information (low, moderate, high, and very high) and Prior (likely versus unlikely intentions), as within-subjects factors (see Fig. 2, and Supplementary analyses).

Then, we aimed to quantify independently the effect of visual evidence and the effect of priors on participant's inference. To do so, and following Skewes et al.'s guidelines (Skewes, Jegindo, \& Gebauer, 2015), we computed two parameters from the signal detection theory framework (Macmillan \& Creelman, 2005). The discriminability parameter $d^{\prime}$ was first estimated within participants from each group, and can be viewed as a measure of how discriminable intentions are within each task (Non-Social and Social tasks). A value of 0 indicates that the subject is unable to discriminate intention A from intention B (e.g., rotating vs. transporting), whereas larger values $(>0)$ indicate a correspondingly greater ability to distinguish between the two competing intentions.

To compare the effect of participants' priors between types of intention (Non-Social vs. Social), a score reflecting this "effect of priors" was calculated for each subject in each task. This score, in turn, was obtained by estimating a "response bias" toward the likely intention (the intention onto which participants had strong priors) using the response criterion parameter $c$. This response criterion was calculated as the sum of the Z-transformed hit rate and the Z-transformed false alarm rate, multiplied by -0.5 (Macmillan \& Creelman, 2005; see also Stanislaw \& Todorov, 1999). Note that the response criterion $c$ measures deviations from the neutral point, where neither response (toward likely or unlikely intentions) is favoured. If the participant's decision criterion is located at this point, $c$ has a value of 0 (no response bias). Negative values of $c$ signify a bias toward responding A (e.g., the likely intention), whereas positive values signify a bias toward the other response $B$ (i.e., the unlikely intention).

We then entered $d^{\prime}$ and response criterion $c$ values into two $2 \times 4 \times 2$ repeated-measures ANOVAs with Amount of visual information (low, moderate, high, and very high) and type of Intention (Non-Social versus Social) as within-subjects factors, and Group (Comparison versus ASD participants) as a between-subjects factor.

The ANOVA on $d$ ' revealed a strong effect of the amount of visual information on discriminability performance: intentions were, as expected, more discriminable as the amount of information increased $(\mathrm{F}(3,108)=222.13, p<0.001, \eta=0.86)$. ASD and comparison participants showed similar discriminability capacities (main effect of Group, $\mathrm{F}(1,36)=3.16, p=0.084$, $\dot{\eta}=0.08$ ), whereas both Non-Social and Social types of intention were equally discriminable (main effect of the type of intention, $F(1,36)=1.66$, $p=0.20$ ). We found a near-threshold interaction effect between the type of intention and the amount of visual information ( $F$ $(3,108)=2.64, p=0.052, \eta=0.068)$, suggesting that Social intentions were overall less well-discriminable than Non-Social intentions, but for high amount of information only (post hoc tests, Non-Social low vs. Social low: ns.; Non-Social MODERATE vs. Social MODERATE: $n s$; Non-Social HIGH vs. Social HIGH: $p=0.003$; Non-Social v. HIGH vs. Social v. HIGH: ns.). The other interaction effects (i.e., Intention-by-Group, Amount of information-by-Group, and Intention-by-Amount-by-Group) were not significant (all Fs $<0.24$, all $P$ 's $>0.86$ ). Interestingly, a complementary analysis showed that ASD' performances tended to improve to a greater extent than comparisons' when increasing the amount of visual information (from low to very high) (main effect: $P=0.07$, $\eta=0.08$; Supplementary Analyses). This improvement was however irrespective of the type of intention (Non-Social, Social).

The ANOVA on response bias (criterion $c$ ) revealed no effect of the group factor (i.e., the magnitude of the response bias was similar across both groups: $\mathrm{F}(1,36)=0.21, p=0.64)$, and no effect of the type of intention (Non-Social vs. Social: $F(1,36)=2.92$, $p=0.097, \eta=0.07)$. Crucially, the interaction effect between group and type of intention was highly significant $(F(1,36)=8.31$, $p=0.006, \eta=0.19)$ : comparison participants showed a greater response bias for the likely intention in the Social, relative to the Non-Social, condition $(P=0.002)$, whereas ASD participants exhibited a response bias of similar magnitude across both conditions $(P=0.42)$. This result suggests that comparison participants relied more on prior information when they had to infer Social intentions relative to Non-Social intentions, whereas ASD participants did not. Consistent with a Bayesian estimation scheme, we found a significant main effect of the amount of visual information 


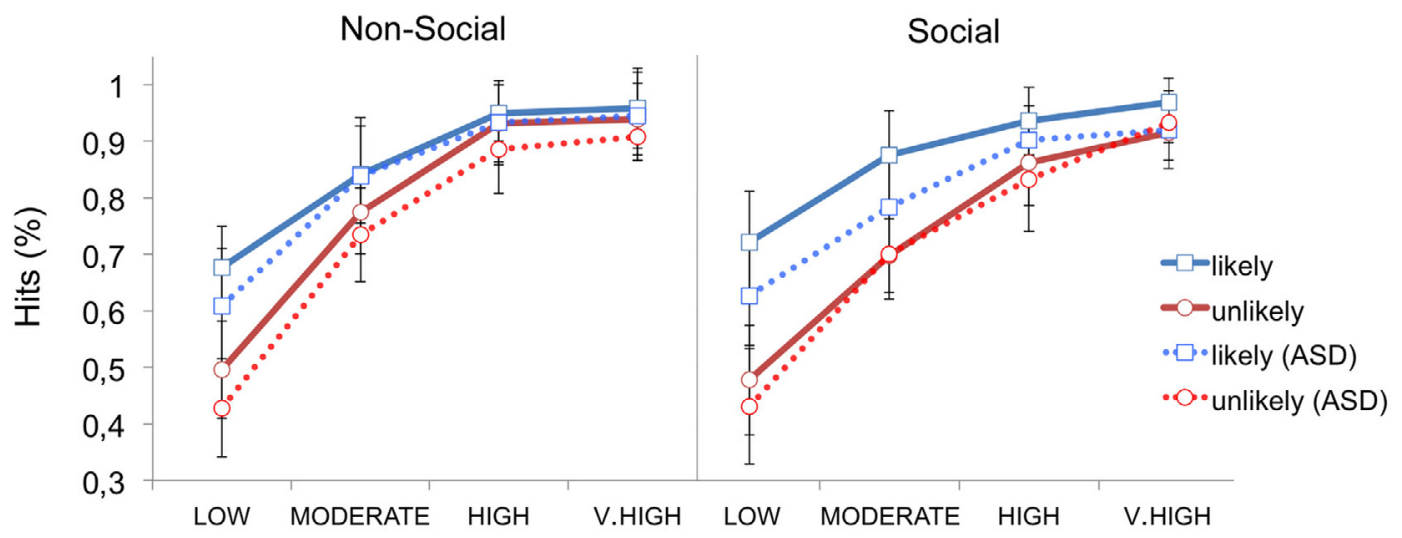

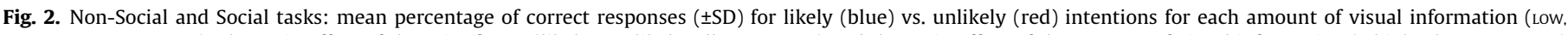

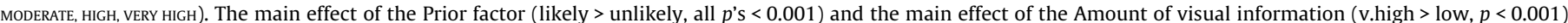

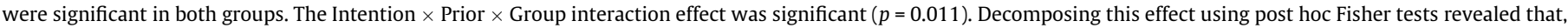

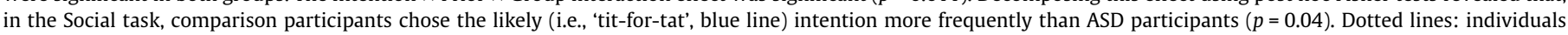

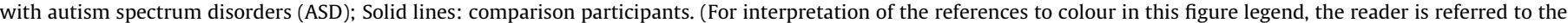
web version of this article.)

$(\mathrm{F}(3,108)=4.50, p=0.005, \eta=0.11)$, with the magnitude of the response bias increasing as the amount of information decreased (see Supplementary Information, Fig. S2). Finally, no significant Intention-by-Amount of visual information, or Group-by-Amount of visual information, or Intention-by-Amount-by-Group, interaction effects, were found (all $F$ 's $(3,108)<0.78$, all $P$ 's $>0.67$ ) (see Fig. 3).

\subsection{Learning effect}

A previous study using the same material and paradigm has shown that typically developed adults exhibit a strong preference for tit-for-tat (TFT) intentions over alternative (non-TFT) intentions (Chambon, Domenech et al., 2011). Importantly, this preference preceded the assignment of any probabilistic bias - i.e., arose early in the task, from the very first trials of the experimental sequence. Specifically, this preference was accounted for by a high proportion of responses toward TFT intentions and by a cost in switching from TFT to non-TFT intentions.

We investigated i) whether this spontaneous preference for TFT intentions was equally present in both groups and ii) whether the social intention and non-social intention priors were similarly learnt in individuals with ASD and comparison participants. We therefore calculated a learning index within each (Non-Social and
Social) task, and directly compared this index between comparison and ASD participants. The learning index was computed by subtracting the response bias in the last block of the sequence from the response bias of the first block of the sequence, in each task separately. For direct comparison between groups and types of intention, this index was input into a $2 \times 2$ repeated-measures ANOVA with Group (Comparison versus ASD) as a betweensubjects factor, and type of Intention (Non-Social versus Social) as a within-subject factor.

A significant Group $\times$ type of Intention interaction was found $(F$ $(1,36)=4.44, p=0.042, \eta=0.11)$, with the magnitude of the response bias increasing more strongly over time in the Social task, relative to the Non-Social task in the ASD group (Fig. 4). Post-hoc analyses revealed that this effect was mainly driven by the fact that, in the comparison group, the response bias increased from the first to the last block of trials in the Non-Social task more than in the Social task $(p=0.059)$. In contrast, no difference was observed between tasks in the ASD group $(p=0.29)$. Note that despite the initial lack of preference for the TFT strategy, the response bias toward TFT responses progressively increased over time in ASD participants, to finally equal that of comparison participants, as revealed by non-significant differences in the response bias for TFT intentions in the last block between the two groups $(p=0.68)$
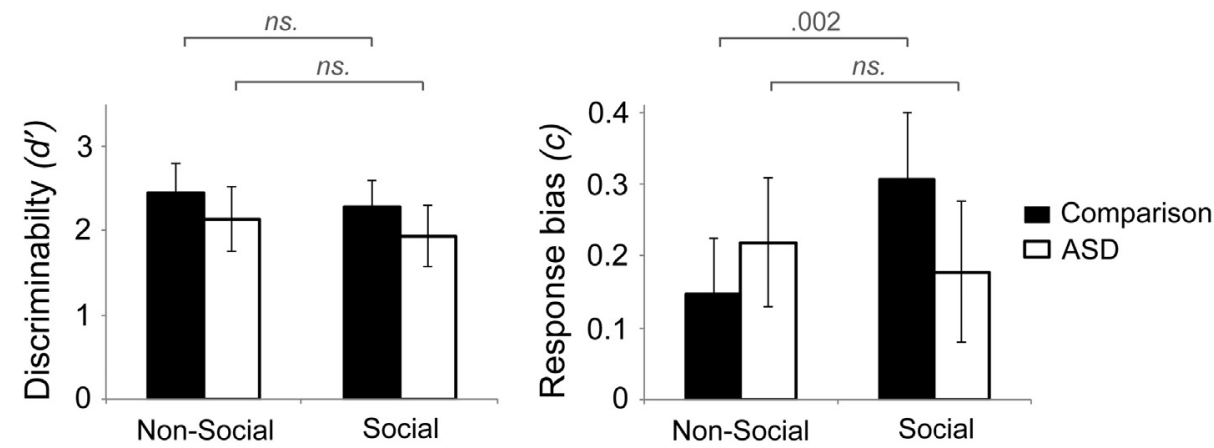

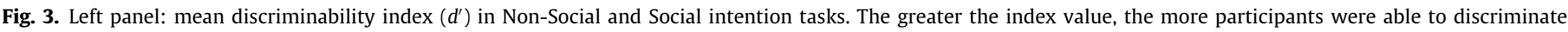

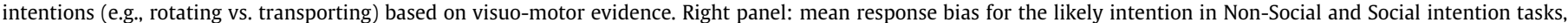

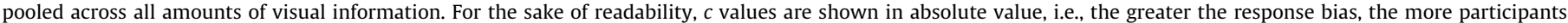
responded toward the likely intention. ns.: non-significant $(>0.05)$. ASD: individuals with autism spectrum disorders. 


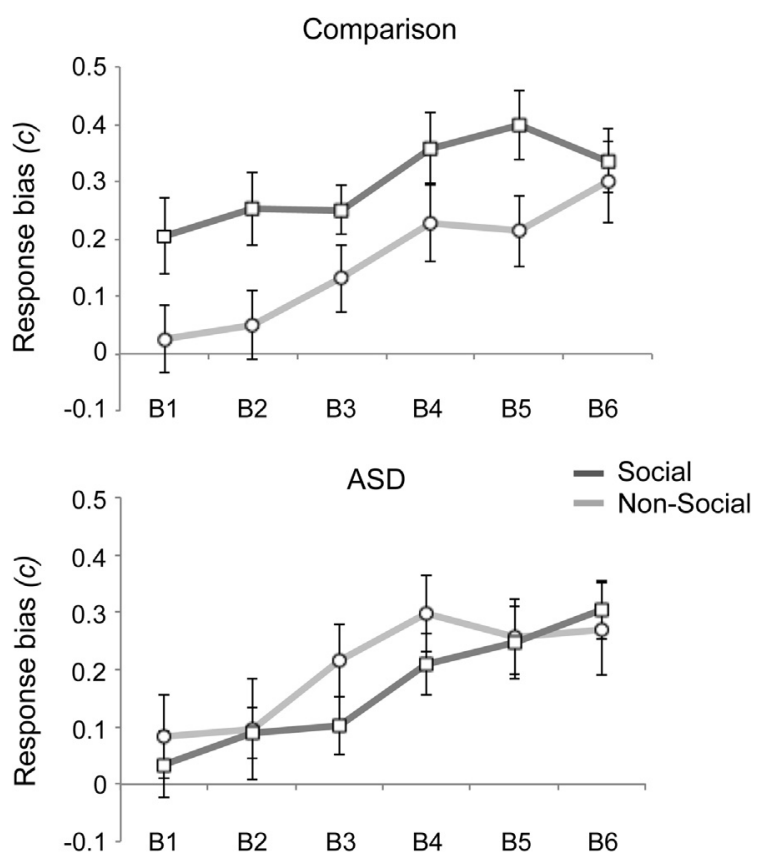

Fig. 4. Response bias (mean $\% \pm \mathrm{SE}$ ) in both comparison and ASD groups throughout the Non-Social and Social intention tasks (from block 1 to block 6). Comparison participants exhibited an early preference for TFT intentions, as illustrated by a significantly greater response bias in the Social (vs. Non-Social) task that is already present from the very first block of trials (B1). ASD participants did not exhibit such an early preference but progressively acquired it throughout the task, as illustrated by a response bias toward TFT intentions that was as large as that of comparison participants at the end of the sequence (B6). For the sake of readability, $c$ values are shown in absolute value, i.e., the greater the response bias, the more participants responded toward the likely intention. $B 1$ to B6: block1 to block 6; ASD: individuals with autism spectrum disorders.

\subsection{Response bias: Correlation analyses}

As mentioned above, the Non-Social and Social tasks mobilize different types of priors. The priors induced in the Non-Social task were purely probabilistic (i.e., they were acquired by sampling the task), whereas the preference for TFT social intentions appears to be present before any probabilistic bias is induced. However, this spontaneous preference gets progressively reinforced over time through selectively increasing the occurrence of a particular intention at the expense of the other competing one.

We speculated that if these two types of priors (probabilistic priors vs. TFT spontaneous preference) do indeed rely on different mechanisms, one should expect the effect of priors, or response bias, in one task to be poorly correlated with the effect of priors, or response bias, in the other task. To test this hypothesis, we performed nonparametric (Spearman's) correlation analyses between response biases calculated for each task in each group of participants, and we compared them using a Fisher's r-to-z transformation test (Weaver and Wuensch, 2013). The response bias in the Non-Social task did not correlate with the response bias in the Social task in comparison participants $(R=0.26, P=0.26)$, whereas they were highly correlated in the ASD group $(R=0.65, P=0.003)$ (Fig. 5). The difference between groups' coefficients was nearthreshold (one-tailed, $p=0.07$ ). The strong correlation found in the ASD group suggests that priors biasing the response of ASD individuals across Social and Non-Social conditions share a similar mechanism. Although exhibiting no inherent preference for TFT intentions, ASD participants would acquire this preference progressively through the extraction of observed regularities (i.e., through probabilistic sampling of the task sequence), unlike comparison participants.

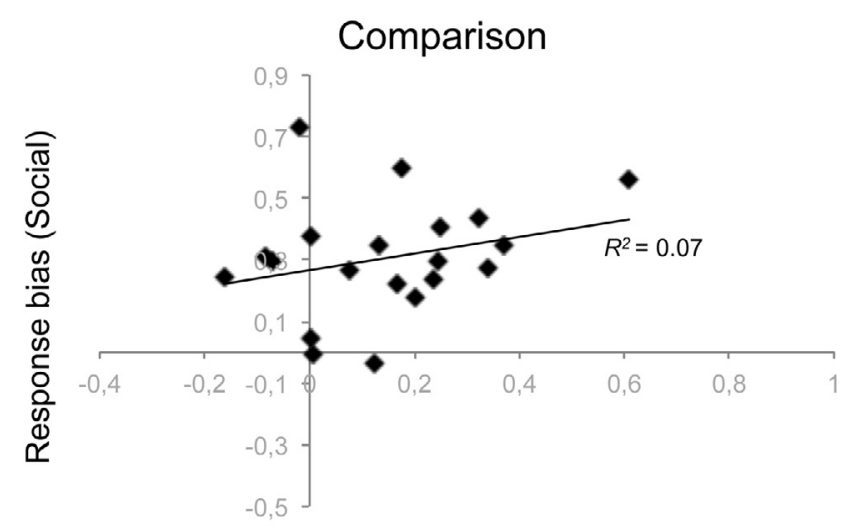

\section{Response bias (Non-Social)}

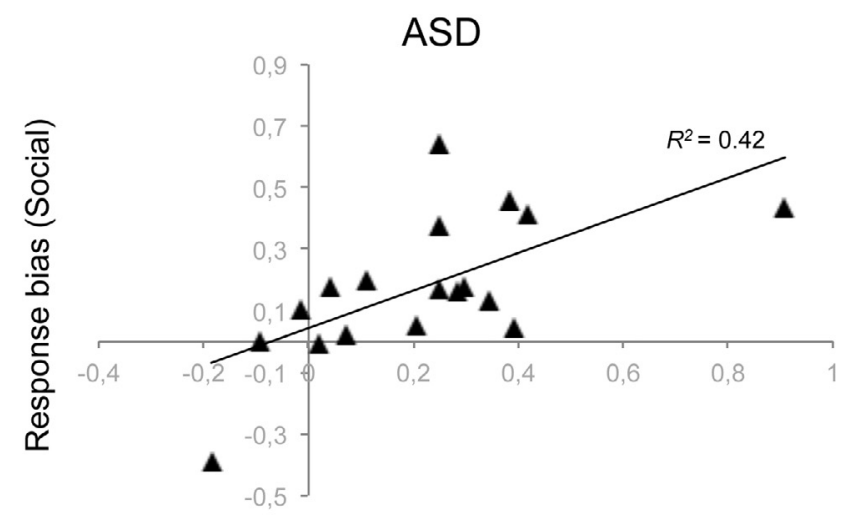

Response bias (Non-Social)

Fig. 5. Correlation analyses between the 'response bias' scores calculated for each task (Social and Non-Social tasks) in each group of participants. The greater the positive correlation, the more likely it is that the response bias toward the likely intention in one task relies on a mechanism similar to the response bias in the other task. ASD: individuals with autism spectrum disorders.

\subsection{Clinical symptoms: Regression analyses}

Regression analyses were conducted to assess whether an abnormal dependence on priors was predictive of the severity of autistic symptoms, in the areas of repetitive behaviours and social interaction as measured by the Autism Diagnostic InterviewRevised (ADI-R) (Lord et al., 1994). For each clinical score, we conducted regression analyses using the 'response bias' score and the 'learning index' as predictor variables. We used either raw scores (simple linear regressions) or their transformed values (simple non-linear regressions with logarithmic, polynomial or exponential transformations). Models with the highest adjusted $R^{2}$ and a $\mathrm{p}<0.05$ are reported.

In the Social task, the response bias significantly and negatively predicted the severity of the autistic symptomatology in the social domain, in the ASD group $\left(R^{2}=0.27, P=0.02\right)$ (Fig. $6 \mathrm{~A}$ ). The lower the propensity to respond toward the likely social intention in ASD participants (i.e. the less they relied on social priors to make their prediction), the higher the severity of the symptoms in the area of reciprocal social interaction, as measured by the ADI subscores. Moreover, the learning index negatively correlated with the ADI sub-scores measuring disturbances in stereotyped and repetitive behaviours $\left(R^{2}=0.24, P=0.03\right.$ ) (Fig. $6 \mathrm{~B}$ ). We did not find any significant correlations between the learning index in the NonSocial condition and the 'stereotypy' $\left(R^{2}=0.15, P=0.12\right)$ or the 'social interaction disorders' $\left(R^{2}=0.16, P=0.10\right)$ dimensions. Note, however, that the negative correlation between social learning 

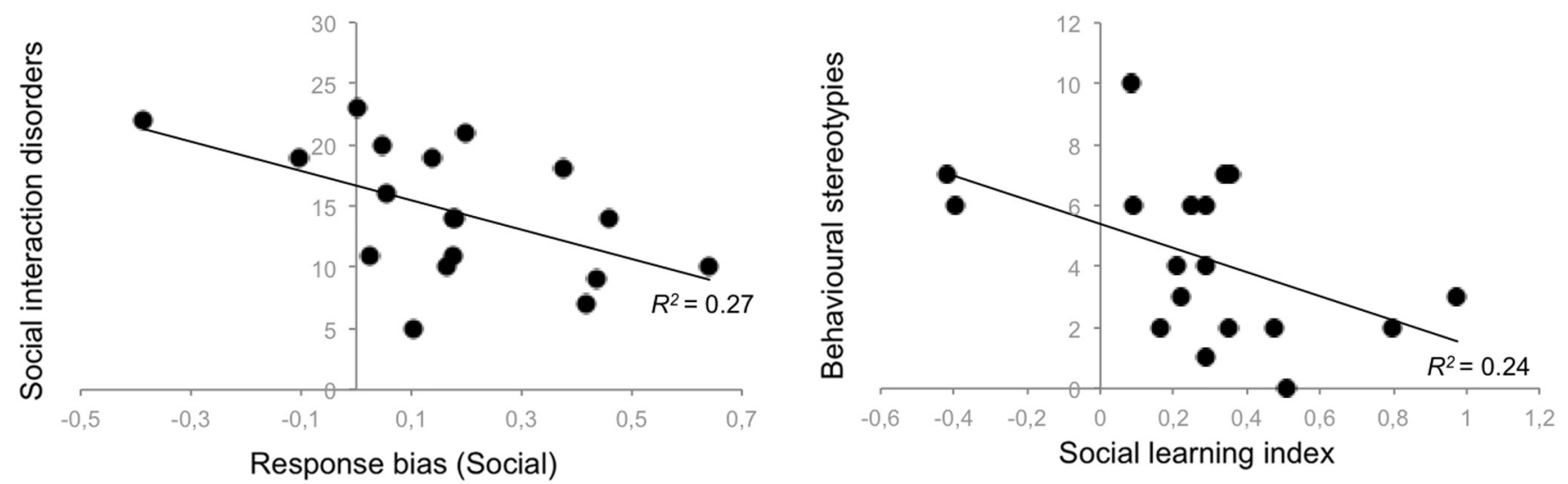

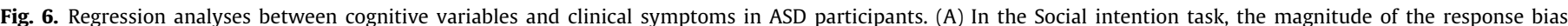

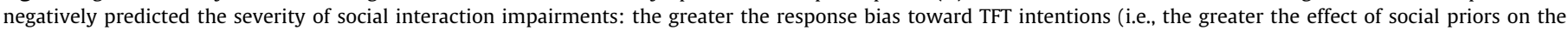

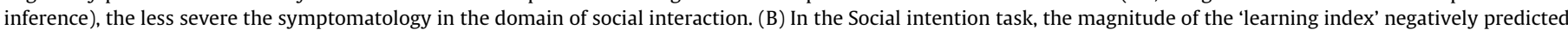

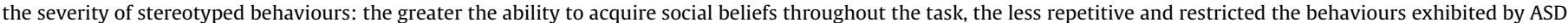
participants.

and stereotypies became only marginally significant when controlling for non-social learning (partial $r$ : $-0.44, p=0.076$ ). This result suggests that ASDs with less pronounced stereotypies were better learners overall - while marginally benefiting from an extra boost when learning from social cues.

\section{Discussion}

The aim of the present study was to investigate whether in adults with ASD poor performance in inferring intentions might be explained by an unbalanced interplay between top-down prior expectations and bottom-up sensory stimulation. Specifically, we expected to find reduced reliance on prior expectations when it comes to inferring social intentions in participants with ASD. We further hypothesized that such reduced dependence, if observed, would correlate with the severity of autistic symptoms in the areas of social interaction and repetitive behaviours.

The current results reveal that both groups were more accurate in recognizing the likely (i.e. biased) intention - i.e., the intention for which the probability of occurrence was set to 0.66 - at the expense of the competing one, and showed increased preference for this intention as the amount of visual information decreased in both Non-Social and Social intention tasks. This finding is in accordance with previous studies (e.g., Chambon, Domenech et al., 2011) and consistent with a Bayesian estimation scheme, which posits that in situations of sparse or incomplete data, the observers tend to compensate for visual uncertainty by appealing to their prior knowledge (Baker et al., 2009; Chambon, Domenech et al., 2011; Jacquet, Chambon, Borghi, \& Tessari, 2012, see Skewes et al., 2015 for a similar result). Our results also show that prior information exerted a greater influence in inferring social motor intentions than non-social motor intentions in comparison participants, while the effect of prior expectations was similar across the two tasks in ASD individuals. Indeed, the magnitude of the response bias was greater in the Social than in the NonSocial task in the comparison group, while this bias exerted a similar influence on ASD group's accuracy in both tasks.

A previous study using the same material and paradigm has shown that typically developed adults exhibit a strong preference for "tit-for-tat" (TFT) social intentions over alternative (non-TFT) intentions, as measured by high proportion of responses toward TFT intentions and by a cost in switching from TFT to non-TFT intentions (Chambon, Domenech et al., 2011). This preference preceded the assignment of any probabilistic bias - i.e., arose early in the task, from the very first trials of the experimental sequence. Remarkably, unlike the comparison group, participants with ASD did not show such initial preference for TFT intentions in the Social task, but they progressively acquired this preference throughout the experiment, so that at the end of the experiment the two groups were equally biased toward predicting TFT, at the expense of non-TFT, intentions.

Note that this reduced, initial sensitivity to social priors cannot be explained by differences in complexity between tasks (e.g., conditional rather than marginal probabilities were to be computed in the social condition) since participants performed at comparable levels across both the social and the non-social experiments. Nor is this result explainable by an increased attentional load in the Social task, resulting from paying attention to a sequence of two actions as opposed to a single act, which would have been more detrimental to ASD than to comparison participants, since both groups were equally successful in recognizing the 'unlikely' intentions in this condition (see Fig. 2, solid and dotted red lines, right panel). Finally, it is of note that difficulties in ASD participants could only be observed in conditions with the lowest amounts of visual information (Low and MODERATE amounts, see Supplementary Analyses, and Fig. 2). This observation is consistent with what would have predicted a Bayesian estimation scheme should the use of priors, rather than the performance itself, be altered. Thus, rather than showing a generalized impairment in inferring other people's intentions (i.e., a general decrease in performance for all types of intention), individuals with ASD showed specific difficulties in predicting 'reciprocating' intentions. In other words, relative to comparison participants, they relied less on the prior belief that people spontaneously converge toward "mirroring" - i.e., toward matching their behavioural responses ('tit-for-tat') - in situations of iterative interactions (André \& Day, 2007; Axelrod, 1997; Chambon, Domenech et al., 2011).

It is noteworthy that the response bias -quantifying the influence of priors on participant's response- in the Non-Social task correlated poorly with the response bias in the Social task in comparison participants, whereas these biases were highly correlated in the ASD group, suggesting that priors influencing the response across Social and Non-Social conditions share a similar mechanism in individuals with ASD. Thus, although exhibiting no inherent preference for TFT intentions, ASD participants would acquire this social preference progressively through the extraction of observed regularities by the same general probabilistic learning mechanism operating in the non-social task. The absence of spontaneous 
preference for the TFT mode of reciprocation - although compensated for by learning throughout the sequence - provides evidence for attenuated social-specific priors in ASD.

Whether perceptual differences in ASD individuals - such as enhanced perceptual functioning (Mottron, Dawson, Soulières, Hubert, \& Burack, 2006), enhanced sensations (Baron-Cohen, Ashwin, Ashwin, Tavassoli, \& Charkrabarti, 2009), or perceptual biases to local or piecemeal processing (Happé \& Frith, 2006) are best explained by a higher weighting of bottom-up sensory evidence ("enhanced sensory precision") or by a weaker influence of top-down priors on perceptual experience ("hypo-priors"), is still a matter of a lively debate (see Brock, 2012; Pellicano \& Burr, 2012; Lawson et al., 2014; Skewes et al., 2015; Van de Cruys et al., 2014). Our results suggest that difficulties with action understanding in ASD individuals might arise from impairments in complementing (i.e. contextualizing) the available sensory evidence with top-down information - i.e., 'prior expectations' about which intention is the most likely cause of what is observed (see Chambon, Domenech et al., 2011; Chambon, Pacherie et al., 2011). Thus, the present study provides one of the first experimental evidence for a markedly reduced influence of top-down priors (as measured by differences in response criterion $c$ ) on the intention inference process, with prior knowledge given less weight in ASD relative to neurotypical participants, and yet normal bottom-up sensory processing (as measured by $d^{\prime}$ ). Importantly, our results are consistent with a number of recent studies showing that ASD present similar discrimination precision as neurotypical participants, but weaker priors in discriminating stimulus orientation features (Skewes et al., 2015), localizing sounds in space (Skewes \& Gebauer, 2016) or predicting communicative actions between agents (von der Lühe et al., 2016), but also reduced adaptation to facial identity (Ewing, Pellicano, \& Rhodes, 2013) and numerosity (Turi et al., 2015) as a result of prior expectations being given less weight in predicting forthcoming stimuli, and more generally with recent theoretical works suggesting reliance on faulty predictive models in individuals with ASD (Sinha et al., 2014; Palmer, Seth, \& Hohwy, 2015).

Noteworthily, the "hypo-priors" hypothesis (Mitchell \& Ropar, 2004; Pellicano \& Burr, 2012) posits that difficulties in using information from the past experience to drive expectations about incoming sensory signals might explain both social and nonsocial symptoms in autism. As mentioned above, attenuated or abnormal (unreliable) top-down prior expectations should lead to greater reliance on bottom-up sensory signals, which might be responsible for enhanced sensation and perception, experience of sensory overload and the often-reported hypersensitivity in ASD. In the absence of priors modulating (i.e., down- or up-regulating) perceptual experience, individuals with ASD would perceive the world more accurately than typically developed individuals. Importantly, it is in uncertain situations that we rely most on our prior beliefs to contextualize and inform our perception (Chambon, Domenech et al., 2011; Lawson et al., 2014). Predicting social events might thus be particularly difficult if one depends on perceptual evidence only, without being able to call on precise prior beliefs to contextualize other people's behaviours (Chambon, Domenech et al., 2011; Chambon, Pacherie et al., 2011). Consistent with this hypothesis, significant differences between the ASD and the comparison groups were found in the social task, in which the many-to-one mappings between causes (i.e., intentions) and observable evidence (i.e., information conveyed by movement kinematics) were increased, making the action scene markedly more uncertain and the relevant computations more difficult.

Impoverished prior knowledge in the social domain, resulting in an incapability to reduce the pervasive complexity of the social world, could make it hard to understand any observed or experi- enced social interactions, and ultimately be responsible for social and motivational disorders often reported in this population. In the Social task, a lack of preference for the 'TFT' mode of interaction predicted the severity of ASD symptomatology in the domain of social interaction, as assessed by the ADI sub-score. This finding strongly suggests that poor expectations in the social domain may also be relevant to explain social difficulties in everyday life. Crucially, the index quantifying the evolution of both social and non-social biases throughout the experiment ("learning index") correlated with the severity of autistic symptomatology in the domain of restricted and repetitive behaviour as measured by the ADI sub-score. Repetitive behaviours and stereotypes can be explained by reduced top-down modulatory control over motor routines and acquired motor plan. An attenuated effect of prior expectations on incoming evidence conveyed by other people's movement kinematics might result in diminished flexibility and generalization leading to repetitive behaviours, such as stimming and self-stimulation, as a means of dealing with the unpredictable nature of other people's actions. Similarly, repetitive behaviours in ASD have been described as attempts to minimize the consequences of environmental unpredictability. Impoverished prior expectations may bring about such unpredictability, and hence repetitive behaviours as a way to mitigate anxiety arising out from unpredictability, i.e., from a "confusing" and "chaotic" world wherein other people's behaviours seem to occur unexpectedly and without cause (Sinha et al., 2014).

Importantly, the reduced ability to make reliable predictions about other's behaviour as a consequence of attenuated topdown priors, is in accordance with a more general disrupted cortical connectivity framework, as a model of ASD neural organization, and neurobiological findings revealing abnormal top-down neuromodulatory control exerted by higher-order regions over posterior occipital and temporal areas responsible for sensory processing (Geschwind \& Levitt, 2007; Just, Cherkassky, Keller, \& Minshew, 2004). Our observation of reduced prior expectations in ASD individuals fits also particularly well with a recent hypothesis suggesting that an underlying impairment in predictive abilities, resulting in an incapability to reduce the intrinsic uncertainty of the world, may account for some of the most salient aspects of the autism phenotype (e.g., sensory hypersensitivites, stereotypies, difficulties with action prediction and Theory of Mind) (Sinha et al., 2014; see also Sevgi, Diaconescu, Tittgemeyer, \& Schilbach, 2016).

Noteworthily, different modes of top-down control can be characterized depending on how "priors" are defined. A distinction can thus be drawn between "empirical priors" that are learnt from past observations, and "systemic priors" that correspond to built-in, and often domain-specific, biases (Clark, 2013; see also Hohwy, Roepstorff, \& Friston, 2008; Trappenberg \& Hollensen, 2013). In the present study, the correlation between the severity of ASD symptomatology in the domain of social interaction and lack of initial preference for the TFT strategy would suggest that social systemic priors are weak or altogether lacking in ASD. Importantly, we observed that ASD individuals were still able to acquire this "systemic" preference though the same general mechanism leading to the acquisition of an "empirical" preference throughout the task. However, this acquisition would be contingent on the severity of repetitive and stereotypic behaviours in ASD individuals, as shown by a negative correlation between behavioural stereotypies and our "learning" index: the less repetitive and restricted the behaviours exhibited by ASD participants, the greater the ability to acquire social beliefs throughout the task.

In the light of these results, we suggest that behavioural stereotypies, as non-functional, suppressible patterns of behaviour, may serve as early clinical intervention targets to improve acquisition of social-specific knowledge in ASD individuals, together with being 
useful indicators for identification and management of vulnerable individuals in the domain of social interactions.

\section{Acknowledgements}

We gratefully acknowledge the commitment of the participants and their families to the pursuit of research in autism. This research was supported by an ANR Grant (ANR-ZR81) to CF, VC, EP and TZ, and by Fondation FondaMental and Fondation de France (00041930) to TZ and ML. VC was supported by a postdoctoral fellowship of the Région Ile-de-France (Paris) and a research grant from the Fyssen Foundation. VC, EP and TZ were supported by ANR-10-LABX-0087 IEC and ANR-10-IDEX-0001-02 PSL*. Designed research: V.C., C.F., E.P., T.Z. Performed research: V.C., P.O.J., M.L. Analyzed data: V.C., C.F., T.Z. Wrote the paper: V.C. C.F., E.P., P.O. J., T.Z. No conflict of interest.

\section{Appendix A. Supplementary material}

Supplementary data associated with this article can be found, in the online version, at http://dx.doi.org/10.1016/j.cognition.2016. 12.005 .

\section{References}

André, J. B., \& Day, T. (2007). Perfect reciprocity is the only evolutionarily stable strategy in the continuous iterated prisoner's dilemma. Journal of Theoretical Biology, 247, 11-22.

Axelrod, R. (1997). The complexity of cooperation: Agent-based models of competition and collaboration. Princeton, New Jersey: Princeton University Press.

Baker, C. L., Saxe, R., \& Tennenbaum, J. B. (2009). Action understanding as inverse planning. Cognition, 113, 329-349.

Baker, C. L., Tenenbaum, J. B., \& Saxe, R. R. (2006). Bayesian models of human action understanding. In Y. Weiss, B. Scholkopf, \& J. Platt (Eds.), Advances in neural information processing systems (pp. 99-106). MIT Press.

Barbalat, G., Chambon, V., Domenech, P., Ody, C., Koechlin, E., Franck, N., \& Farrer, C. (2011). Impaired hierarchical control within the lateral prefrontal cortex in schizophrenia. Biological Psychiatry, 70, 73-80.

Barbalat, G., Chambon, V., Franck, N., Koechlin, E., \& Farrer, C. (2009). Organization of cognitive control within lateral prefrontal cortex in schizophrenia. Archives of General Psychiatry, 66, 1-10.

Baron-Cohen, S. (1995). Mindblindness. An essay on autism and theory of mind. Cambridge: MIT Press.

Baron-Cohen, S., Ashwin, E., Ashwin, C., Tavassoli, T., \& Charkrabarti, B. (2009) Talent in autism: Hyper-systemizing, hyper-attention to detail and sensory hypersensitivity. Philosophical Transactions of the Royal Society of London. Series B, Biological Sciences, 364, 1377-1383.

Baron-Cohen, S., Leslie, A. M., \& Frith, U. (1985). Does the autistic child have a 'theory of mind'? Cognition, 21(1), 37-46.

Baron-Cohen, S., Leslie, A. M., \& Frith, U. (1986). Mechanical, behavioural and intentional understanding of picture stories in autistic children. Brit J Dev Psychol, 4, 113-125.

Brock, J. (2012). Alternative Bayesian accounts of autistic perception: Comment on Pellicano and Burr. Trends in Cognitive Sciences, 16(12), 573-574.

Cattaneo, L., Fabbri-Destro, M., Boria, S., Pieraccini, C., Monti, A., Cossu, G., \& Rizzolatti, G. (2007). Impairment of actions chains in autism and its possible role in intention understanding. Proc Natl Acad Sci USA, 104(45), 17825-17830.

Chambon, V., Domenech, P., Barbalat, G., Pacherie, E., Jacquet, P. O., \& Farrer, C. (2012). Reply: The Bayesian equation and psychosis. Brain, 135, e218.

Chambon, V., Domenech, P., Pacherie, E., Koechlin, E., Baraduc, P., \& Farrer, C. (2011). What are they up to? The role of sensory evidence and prior knowledge in action understanding. PLOS ONE, 6, e17133.

Chambon, V., Pacherie, E., Barbalat, G., Jacquet, P., Franck, N., \& Farrer, C. (2011). Mentalizing under influence: Abnormal dependence on prior expectations in patients with schizophrenia. Brain, 134, 3725-3738.

Clark, A. (2013). Whatever next? Predictive brains, situated agents, and the future of cognitive science. Behav Brain Sci, 36(3), 181-204.

Ewing, L., Pellicano, E., \& Rhodes, G. (2013). Atypical updating of face representations with experience in children with autism. Dev Sci, 6(1), 116-123.

Fabbri-Destro, M., Cattaneo, L., Boria, S., \& Rizzolatti, G. (2009). Planning actions in autism. Experimental Brain Research, 192(3), 521-525.

Fletcher, P. C., \& Frith, C. D. (2009). Perceiving is believing: A Bayesian approach to explaining the positive symptoms of schizophrenia. Nature Reviews Neuroscience, 10, 48-58.

Frith, U. (1989). Autism: Explaining the enigma. Oxford: Basil Blackwell.

Geschwind, D. H., \& Levitt, P. (2007). Autism spectrum disorders: Developmental disconnection syndromes. Current Opinion in Neurobiology, 17, 103-111.
Gillberg, C., Gillberg, C., Råstam, M., \& Wentz, E. (2001). The Asperger Syndrome (and high-functioning autism) Diagnostic Interview (ASDI): A preliminary study of a new structured clinical interview. Autism, 5, 57-66.

Gomot, M., \& Wicker, B. (2012). A challenging, unpredictable world for people with autism spectrum disorder. International Journal of Psychophysiology, 83(2), 240-247.

Hamilton, A. (2009). Goals, intentions and mental states: Challenges for theories of autism. Journal of Child Psychology and Psychiatry, 50(8), 881-892.

Happé, F., \& Frith, U. (1996). The neuropsychology of autism. Brain, 119(4), 1377-1400.

Happé, F., \& Frith, U. (2006). The weak coherence account: Detail-focused cognitive style in autism spectrum disorders. Journal of Autism and Developmental Disorders, 36, 5-25.

Hohwy, J., Roepstorff, A., \& Friston, K. (2008). Predictive coding explains binocular rivalry: An epistemological review. Cognition, 108(3), 687-701.

Hughes, C. (1996). Brief report: Planning problems in autism at the level of motor control. Journal of Autism and Developmental Disorders, 26(1), 99-107.

Jacquet, P., Chambon, V., Borghi, A. M., \& Tessari, A. (2012). Object affordances tune observers' prior expectations about tool-use behaviors. PLoS ONE, 7(6), e39629.

Just, M., Cherkassky, V., Keller, T., \& Minshew, N. (2004). Cortical activation and synchronization during sentence comprehension in high-functioning autism: Evidence of underconnectivity. Brain, 127, 1811-1821.

Kilner, J. M. (2011). More than one pathway to action understanding. Trends in Cognitive Sciences, 15, 352-357.

Kilner, J. M., Friston, K. J., \& Frith, C. D. (2007a). Predictive coding: An account of the mirror neuron system. Cognitive Processing, 8, 159-166.

Kilner, J. M., Friston, K. J., \& Frith, C. D. (2007b). The mirror-neuron system: A Bayesian perspective. NeuroReport, 18, 619-623.

Lawson, R. P., Rees, G., \& Friston, K. J. (2014). An aberrant precision account of autism. Frontiers in Human Neuroscience, 8, 302.

Lord, C., Risi, S., Lambrecht, L., Cook, E. H., Jr., Leventhal, B. L., DiLavore, P. C., \& Rutter, M. (2000). The autism diagnostic observation schedule-generic: A standard measure of social and communication deficits associated with the spectrum of autism. Journal of Autism and Developmental Disorders, 30, 205-223.

Lord, C., Rutter, M., \& Le Couteur, A. (1994). Autism diagnostic interview-revised: A revised version of the diagnostic Interview for caregivers of individuals with possible pervasive developmental disorders. Journal of Autism and Developmental Disorders, 24, 659-685.

Macmillan, N. A., \& Creelman, C. D. (2005). Detection theory: A user's guide (2nd ed.). Mahwah, NJ: Lawrence Erlbaum Associates.

McKay, R. (2012). Delusional inference. Mind \& Language, 27, 330-355.

Mitchell, P., \& Ropar, D. (2004). Visuo-spatial abilities in autism: A review. Infant and Child Development, 13, 185-198.

Mottron, L., Dawson, M., Soulières, I., Hubert, B., \& Burack, J. (2006). Enhanced perceptual functioning in autism: An update, and eight principles of autistic perception. Journal of Autism and Developmental Disorders, 36(1), 27-43.

Palmer, C. J. Seth, A. K. \& Hohwy, J. (2015). The felt presence of other minds: Predictive processing, counterfactual predictions, and mentalising in autism. Consciousness \& Cognition, 36, 376-389.

Pellicano, E., \& Burr, D. (2012). When the world becomes 'too real': A Bayesian explanation of autistic perception. Trends in Cognitive Sciences, 16(10), 504-510.

Russell, J., \& Jarrold, C. (1998). Error-correction problems in autism: Evidence for a monitoring impairment? Journal of Autism and Developmental Disorders, 28, 45-61.

Russell, J., \& Jarrold, C. (1999). Memory for actions in children with autism: Self versus other. Cognitive Neuropsychiatry, 4, 303-331.

Sevgi, M., Diaconescu, A. O., Tittgemeyer, M., \& Schilbach, L. (2016). Social bayes: Using Bayesian modeling to study autistic trait-related differences in social cognition. Biological Psychiatry, 80, 112-119.

Sinha, P., Kjelgaard, M. M., Gandhi, T. K., Tsourides, K., Cardinaux, A. L., Pantazis, D., ... Held, R. M. (2014). Autism as a disorder of prediction. Proc Natl Acad Sci USA, 111(42), 15220-15225.

Skewes, J. C., \& Gebauer, L. (2016). Suboptimal auditory localization in autism spectrum disorder: Support for the Bayesian account of sensory symptoms. Journal of Autism and Developmental Disorders, 46(7), 2539-2547.

Skewes, J. C., Jegindo, E. M., \& Gebauer, L. (2015). Perceptual inference and autistic traits. Autism, 19, 301-307.

Smith, I. M., \& Bryson, S. E. (1994). Imitation and action in autism: a critical review. Psychological Bulletin, 116, 259-273.

Stanislaw, H., \& Todorov, N. (1999). Calculation of signal detection theory measures. Behavior Research Methods, Instruments, E Computers, 31, 137-149.

Trappenberg, T., \& Hollensen, P. (2013). Sparse coding and challenges for Bayesian models of the brain. Behavioral and Brain Sciences, 36(3), 232-233.

Turi, M., Burr, D. C., Igliozzi, R., Aagten-Murphy, D., Muratori, F., \& Pellicano, E. (2015). Children with autism spectrum disorder show reduced adaptation to number. Proceedings of the National Academy of Sciences USA, 112(25), 7868-7872.

Van de Cruys, S., Evers, K., Van der Hallen, R., Van Eylen, L., Boets, B., de-Wit, L., \& Wagemans, J. (2014). Precise minds in uncertain worlds: Predictive coding in autism. Psychological Review, 121(4), 649-675.

Vanvuchelen, M., Roeyers, H., \& De Weerdt, W. (2007). Nature of motor imitation problems in school-aged males with autism: How congruent are the error types? Developmental Medicine and Child Neurology, 49, 6-12.

von der Lühe, T., Manera, V., Barisic, I., Becchio, C., Vogeley, K., \& Schilbach, L. (2016). Interpersonal predictive coding, not action perception, is impaired in autism. Philosophical Transactions of the Royal Society of London. Series B, Biological Sciences, 371, 20150373. 
von Hofsten, C., \& Rosander, K. (2012). Perception-action in children with ASD. Frontiers in Integrative Neuroscience, 12, 115.

Weaver, B., \& Wuensch, K. L. (2013). SPSS and SAS programs for comparing Pearson correlations and OLS regression coefficients. Behavior Research Methods, 3, 880-895.

Wechsler, D. (1999). Wechsler abbreviated scale of intelligence. Psychological Corporation.

Zalla, T., Bouchilloux, N., Labruyere, N., Georgieff, N., Bougerol, T., \& Franck, N. (2006). Impairment in event sequencing in disorganised and non-disorganised patients with schizophrenia. Brain Research Bulletin, 68(4), 195-202.
Zalla, T., Labruyere, N., Clément, A., \& Georgieff, N. (2010). Predicting ensuing actions in children and adolescents with autism spectrum disorders. Experimental Brain Research, 201(4), 809-819.

Zalla, T., Labruyere, N., \& Georgieff, N. (2006). Goal-directed action representation in autism. Journal of Autism and Developmental Disorders, 36(4), 527-540.

Zalla, T., Labruyère, N., \& Georgieff, N. (2013). Representing actions and goals in individuals with Autism Spectrum Disorders. Journal of Autism and Developmental Disorders, 43(10), 2353-2365.

Zalla, T., Verlut, I., Franck, N., Puzenat, D., \& Sirigu, A. (2004). Perception of dynamic action in patients with schizophrenia. Psychiatry Research, 128, 39-51. 